\title{
LO SCHEMA ONTO-EPISTEMICO DELLA LINEA
}

\section{L'importanza del contesto}

Credo che sia importante non dimenticare il contesto in cui si colloca la Linea. Siamo all'interno di una trattazione che occupa i libri VI e VII della Repubblica, che «costituiscono in primo luogo una lunga appendice destinata ad argomentare tanto sul piano etico quanto su quello onto-epistemologico, la legittimità e la plausibilità della proposta di un governo dei filosofi» ${ }^{1}$, con una sequenza di immagini: il Sole, la Linea, la Caverna. Si tratta di un testo compatto: per giustificare la terza ondata, Socrate deve chiarire cosa intende per filosofi e per filosofia. Ciò spiega il rilievo che hanno questi testi per la comprensione del pensiero di Platone. Non si può, quindi, ignorare il contesto, anche perché la Linea costituisce l'anello di congiunzione tra le altre due immagini.

Tuttavia, data la natura di questo scritto, che ha un tema specifico e limiti di spazio, proporrò, in forma schematica, solo gli elementi attinenti alla tesi che vorrei illustrare ${ }^{2}$, rinunciando a completare la trattazione con la Caverna ${ }^{3}$.

${ }^{1}$ M. VegETTI, Introduzione, in ID. (a cura di), Platone. La Repubblica, Libri VI-VII, v, Napoli 2003, p. 13.

${ }^{2}$ Per lo stesso motivo non affronterò il dibattito che, su quasi tutti i punti, divide gli studiosi, né mi impegnerò a contrastare tesi che non mi paiono convincenti.

${ }^{3}$ Rimando a M. MigLIORI, Sul Bene. Materiali per una lettura unitaria dei dialoghi e 


\subsection{Presentazione del filosofo}

Il tema è introdotto alla fine del libro $\mathrm{V}$, con una prima delineazione del filosofo ${ }^{4}$. Schematicamente, questi mi sembrano i punti di maggior rilievo:

1. il filosofo privilegia il tutto e l'intero, non la parte ( $474 \mathrm{C}$; $475 \mathrm{~B}-\mathrm{C})$;

2. Platone contrappone chi si limita a fruire delle cose belle a chi ama la verità e le cose in sé (475 E, 476 B-D);

3. su questa base si stabilisce una prima distinzione, tra un pensiero

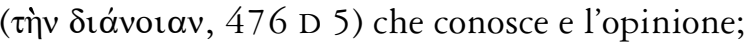

4. Platone fonda tale distinzione sul piano ontologico:

4.1.1. contrappone essere e non essere ${ }^{5}$ : «solo ciò che è in senso pieno è pienamente conoscibile, mentre ciò che non è è del tutto inconoscibile» (477 A 3-4);

4.1.2. la doppia radicale opposizione, essere-non essere / conoscibileinconoscibile, porta a uno schema trinario, con in mezzo ciò che è e insieme non è;

4.2. l'analoga forma di conoscenza, intermedia tra ignoranza e scienza, è la doxa (477 A-B, 478 C);

4.3. pertanto, è facile distinguere opinione e scienza, sempre designate come doxa ed episteme:

delle testimonianze indirette, in G. REALE-S. SCOLNICOV (eds), New Images of P lato. Dialogues on the Idea of the Good, Sankt Augustin 2002, pp. 115-49.

${ }^{4}$ Cfr. F. Ferrari, Teoria delle idee e ontologia, in M. VegetTi (a cura di), Platone. La Repubblica, Libro V, IV, Napoli 2000, pp. 365-92, p. 365.

5 «Scorrendo la più recente bibliografia relativa a questa sezione, si viene a scoprire che pressoché tutti i sensi che il verbo essere può assumere in greco sono stati attribuiti a Platone» (F. FERRARI, Teoria cit., p. 377), quello esistenziale, quello predicativo e quello veritativo-proposizionale. Questo dato è significativo: credo che tali distinzioni siano "imposte" ad un contesto che non le aveva, o che non dava loro il valore che noi gli attribuiamo. Platone si muove in una situazione "anteriore" a queste distinzioni, per cui le diverse accezioni facilmente si richiamano e convivono. Come mostra Ferrari, nello stesso testo è possibile individuare, in successione, "essere" con valore predicativo ed esistenziale, senza particolari stacchi; di conseguenza, anche la presenza di un valore predicativo non esclude sempre il significato esistenziale, ma comporta «che tale significato o sia già contenuto nel contesto dell'argomentazione [...] oppure che sia incorporato nel senso predicativo del verbo» (ivi, p. 379). 
4.3.1. per il loro potere: la doxa non ha il potere della scienza ( 477 B); opinione e scienza (la più potente di tutte le facoltà, 477 D 9) sono distinte, perché non si può confondere un sapere infallibile con uno che non lo è ( $477 \mathrm{E})$;

4.3.2. per l'oggetto: la scienza si occupa di ciò che è ( 477 B, 478 A); l'oggetto della doxa è la realtà che insieme è e non è, mentre quello dell'altra sono le realtà che sono sempre in sé immodificate (479 E).

Il discorso ha qui acquisito una improvvisa stabilità terminologica ed «episteme acquisisce qui per la prima volta nel dialogo [corsivo mio] - nel contesto dell'opposizione gnoseologica con doxa e di quella ontologica fra essere e divenire - il valore forte di conoscenza stabile e veritiera» ${ }^{6}$. Questo è l'elemento che qualifica e distingue il filosofo.

Inoltre, la doxa, ripetutamente qualificata come intermedia $(\mu \varepsilon \tau \alpha \xi \hat{v})^{7}$, non viene del tutto condannata. Infatti la conoscenza delle cose belle non viene denigrata in quanto tale, ma - ogni volta si ribadisce - perché i filodossi si limitano a tali cose e non conoscono l'Idea che le fa essere tali ${ }^{8}$; di converso ciò che qualifica il sapere del filosofo è proprio questa superiore conoscenza ${ }^{9}$. Quindi la doxa, pur avendo oggetti privi della stabilità necessaria per una vera conoscenza, difetta soprattutto per la mancanza dell'altro più rilevante dato. Certo, lo schema proposto non è dialettico e ogni momento

${ }^{6}$ M. VegetTi (a cura di), Platone. La Repubblica, IV, cit., p. 96 nota 127. Sono in disaccordo con quegli interpreti che vedono qui una rottura "drammatica", un radicale cambiamento del ruolo di Glaucone, che comporterebbe la svalutazione della parte finale del libro V, che avrebbe solo una funzione pedagogica e non filosofica. Tralasciando la questione se c'è qualcosa, nei dialoghi, che non sia insieme filosofico e "pedagogico", ha ragione F. FERRARI, Teoria cit., p. 368, a sottolineare che «un attento esame dello sviluppo drammatico e della sequenza argomentativa del testo non sembra, tuttavia, legittimare un'interpretazione di questo tipo». Infatti «i teoremi a favore dei quali Socrate argomenta [...] non sono diversi da quelli presentati in forma assertiva nella prima parte della sezione. In verità, si tratta della medesima posizione filosofica» (ivi, p. 369).

8,9 .

7477 A 7, 8, 10 (due volte), 478 D 3, 6, 8, 11, 478 E 5 (due volte), 479 C 7, 479 D 4,

${ }^{8}$ Cfr. 476 B, C, 479 A, E, 480 A.

${ }^{9}$ Cfr. 476 B, C-D, $479 \mathrm{E}$. 
appare come isolato, ma - dato il modello triadico - la totale contrapposizione che c'è tra sapere e ignoranza (come tra essere e non essere) tra doxa ed episteme non c'è.

Infine, il ragionamento muove da uno schema trinario, ma porta ad una conclusione binaria, alla netta distinzione tra filodossi e filo-sofi $(480 \mathrm{~A})$. Si apre la possibilità di due diverse trattazioni, entrambe presenti in Platone: nel modello triadico abbiamo la contrapposizione radicale essere-non essere, sapere-ignoranza, con una posizione intermedia che non è il nulla sul piano ontologico e non è ignoranza sul piano conoscitivo: l'opinione non ha la forza della scienza ma è, in qualche modo, un sapere. Nel modello binario, data la contrapposizione doxa-episteme, bisogna dire che i fenomeni non hanno vera realtà e che la doxa non conosce davvero le cose su cui opina ( $479 \mathrm{E})$.

\subsection{La ripresa del tema nel libro VI}

Alla fine del libro $v$, Platone ha stabilito alcuni parametri con cui affrontare nel VI «ciò che ne consegue» (484 B 3) in relazione al tema, subito ricordato, della guida della città. La necessità di isolare la figura del filosofo, l'unico che può salvare lo Stato, comporta una radicale contrapposizione tra filosofi e filodossi, con un duro attacco alla doxa. Infatti, per "agevolare" la scelta tra le due figure con un esempio, si pongono in alternativa una sentinella dalla vista acuta e una cieca (484 C-D). Questo stacco della figura del filosofo viene rafforzato 1$)$ con il tema della verità, su cui si era già richiamata l'attenzione (v 475 E 4), ma che viene con maggiore forza riproposto ${ }^{10}$, fino a dire che il filosofo è amico e congenere della verità

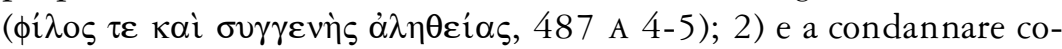
loro che sono privi di una conoscenza stabile (non hanno nell'anima un modello né possono guardare la somma verità ${ }^{11}$, contemplando-

${ }^{10}$ Cfr. 485 C, D, 486 D, 490 A, C, 499 A, 501 D.

${ }^{11}$ M. VegetTi (a cura di), Platone. La Repubblica, v, cit., p. 37 nota 9: «un probabile riferimento all'idea del buono, non menzionata esplicitamente perché non ancora introdotta nella discussione: cfr. VII 540a8 sg., dove si dice più esplicitamente dei dialettici che "quando abbiano visto il buono in sé e siano in grado di valersene come modello 
la nel modo più esatto possibile, così da stabilire i canoni relativi alle cose belle, giuste, buone) (484 C-D).

Nella successiva trattazione, emergono alcuni dati rilevanti per il nostro discorso:

1. si riafferma che il filosofo conosce la realtà che è e che è stabile (484 B, D, 485 B, 486 D 10);

2. tali realtà sono ordinate razionalmente, costituiscono una realtà divina e ordinata (500 C 9; cfr. 500 E 3) che il filosofo cerca di imitare divenendo divino e ordinato per quanto possibile ad un essere umano

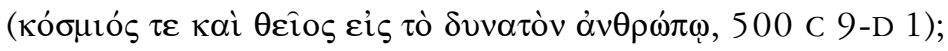

3. si riafferma l'attenzione per il tutto e l'intero: anche se si rivolge a

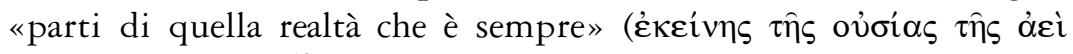
oüбns, 485 в 1-2) ${ }^{12}$, subito si ribadisce che il filosofo ama tutta quella realtà stabile ( 485 B 5) senza trascurarne alcuna parte; egli aspira all'intero e al tutto nell'ambito divino e umano (486 A 5-6);

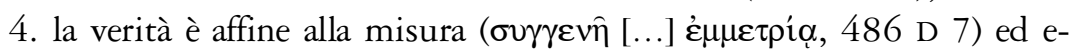

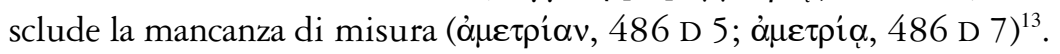

Come si vede, il testo getta un ponte verso il vil libro, ma nel contempo sottolinea che la relazione con i principi deve essere cercata per quanto è possibile. Platone afferma da una parte (in sê) la necessaria relazione con una realtà somma, senza la quale non si dà nulla di vero, giusto etc. ${ }^{14}$, dall'altra (per noi) i limiti della conoscenza umana. Quindi, da una parte, "dimenticando" lo schema triadico e l'ignoranza, accentua la contrapposizione episteme-doxa, dall'altra dice che l'episteme, scienza massima, va sempre accompagnata dall'aggettivo "umana".

( $\pi \alpha \rho \alpha \delta \varepsilon i \gamma \mu \alpha \tau \iota ~ \chi \rho \omega \mu \varepsilon ́ v o v \varsigma) "$ potranno ordinare la città e se stessi».

${ }^{12}$ Cfr. ivi, p. 38 nota 13: «il genitivo chiarisce come il sapere filosofico renda ogni volta visibile una "parte" del campo delle essenze immutabili e non lo riveli immediatamente nella sua totalità».

${ }^{13}$ Questo legame è importante, in quanto credo che qui si trovi la radice della "somiglianza" tra verità e Bene cui subito dopo Platone allude. Questo, naturalmente, se è possibile sostenere che il Bene è Misura...

${ }^{14}$ Come verrà poi detto esplicitamente, VI 505 A-B. 
Platone ci offre infine una sintesi sulla natura del filosofo, una difesa perfettamente adeguata ( $\mu \varepsilon \tau \rho i \omega \varsigma, 490$ A 8; $\mu \varepsilon \tau \rho i \omega ́ \tau \alpha \tau \alpha, 490$ B 8): «colui che veramente ama la conoscenza è per natura pronto a combattere per raggiungere ciò che è, e non si ferma alle molte singole cose che sono oggetto dell'opinione, ma procede senza scoraggiarsi e non smette di amare prima di aver colto la natura di ciascuna cosa che è, con la parte dell'anima cui spetta coglierla - la coglie in quanto è congenere - con questa avvicinandosi e unendosi a ciò che realmente è, generando intelligenza e verità» (490 A 8-B 6).

C'è quindi una processualità che parte dalle singole cose, una conoscenza che non ignora l'empirico ma che procede oltre, salvando l'intero. Infatti Socrate ha precisato che il filosofo, pur accentrando la sua attenzione sulle realtà stabili, non ha meno esperienza

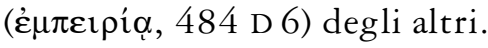

\subsection{Il Bene}

Questa trattazione culmina nella conoscenza massima ( $\tau \grave{\alpha}$ $\mu \varepsilon \dot{\gamma} \gamma \sigma \tau \alpha \mu \alpha \theta \eta \dot{\mu} \mu \alpha \tau)$, che - si chiarisce subito senza alcuna giustificazione teorica - è quella del Bene (505 A).

L'inserimento di questa conoscenza serve, prima di tutto, per condannare la precedente scelta (IV 435 D) di evitare un discorso più lungo. Agli altri interlocutori la trattazione era apparsa misurata ( $\mu \varepsilon \tau$ í $\omega \varsigma, 504$ в 8), ma Socrate obietta: «una misura di tali cose se lascia fuori una parte qualsiasi dell'essere non è certo misurata ( $\left.\mu \varepsilon \tau \tau^{\prime} \omega \varsigma\right)$. Infatti una realtà imperfetta non è misura ( $\left.\mu \varepsilon^{\prime} \tau \rho o v\right)$ di nulla» (504 C 1-2).

Un filosofo non può accontentarsi se vuole venire a capo di quella disciplina che è la più sublime e la più adatta a lui $(504 \mathrm{D}) \mathrm{e}$ che richiede il massimo rigore $(504 \mathrm{E})$. Così si esplicita la ragione di fondo di questa lunga trattazione che, a partire dalla fine del libro $\mathrm{V}$, impeg na tutto il VI e il vII.

Non possiamo ovviamente affrontare il tema del Bene. Possiamo però sottolineare il procedimento con cui Platone ha complessificato il discorso chiarendone la natura verticale: in questo suo "andare oltre", il filosofo è passato dalla dimensione empirica a quella delle Idee a quella di una realtà ad un tempo necessaria ed 
estremamente difficile da cogliere in modo adeguato. Questo è il dato di fondo: da una parte si può dire che non abbiamo conoscenza adeguata del Bene, dall'altro che, se non lo conosciamo (ov̉ $\chi$

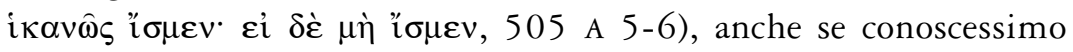
benissimo tutto il resto, nulla sarebbe più utile perché non potremmo sapere se e quando è buono $(505 \mathrm{~A})$. La cosa viene ribadita: malgrado la difficoltà, si esclude che i reggitori possano restare all'oscuro di un oggetto così importante, anzi devono averne scienza (505 D-506 B).

Quindi, non c'è una contrapposizione: c'è il problema della conoscenza adeguata del Bene, ma Platone afferma che dobbiamo averne conoscenza senza però qualificare come sia tale conoscenza.

Conferma questa "oggettiva" ambivalenza il fatto che Socrate da una parte riafferma la sua ignoranza e oppone resistenza, dall'altra parla della possibilità di sentire da altri «cose luminose e belle» (506 D 1) sul tema e, soprattutto, procede, non parlando del Bene ma dandone una delineazione così ricca da risultare possibile solo sulla base di un vero sapere. La cosa non deve stupire: Socrate dice che Adimanto ha già sentito questi discorsi molte volte $(504 \mathrm{E} 8$; 505 A 3) e infatti sa già abbastanza (505 A 4) quello che Socrate vuole dire.

Aggiungo schematicamente alcuni elementi di questa importante trattazione:

1.1. da una parte si afferma la processualità: Socrate parte dalle molte cose belle e buone, che vengono distinte nel discorso razionale; poi si afferma il bello in sé e il buono in sé, cioè un sistema di singole Idee, principio unitario della molteplicità sottostante $(507 \mathrm{~B})$;

1.2. dall'altra si afferma lo stacco: le Idee sono pensate e non viste, le cose sono viste e non pensate (ibid.);

2. il Sole consente una ricca analogia:

2.1. Si parte dal rapporto Bene: intelletto/intelligibili = sole : vista/oggetti (508 B-C). Il sole, che non è la vista, anche se è l'organo

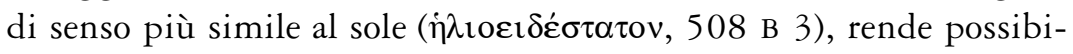
le, anzi causa ( $\alpha i ̈ \tau 1 o \zeta, 508$ B 9) la vista; proprio in quanto causa può essere colto dalla vista. La causalità del sole in un senso riguarda gli 
oggetti illuminati, in un altro riguarda la facoltà ( B 6) che l'occhio possiede. Analogamente l'anima, quando guarda ciò

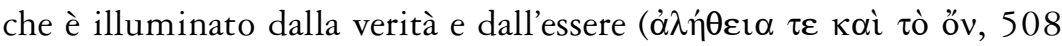
D 5), lo conosce e appare dotata di intelligenza (vôิv ع̌ $\chi \varepsilon v v, 508$ D 6). Il parallelo regge perché la verità svolge la funzione della luce ${ }^{15}$. 2.2. Infatti Platone richiama la centralità dell'Idea del Bene che è $a$ monte di verità ed essere: essa

2.2.1. «conferisce la verità alle cose conosciute e dà la facoltà a chi conosce» (508 E 1-2; cfr. 509 A 6-7);

2.2.2. «essendo causa della scienza e della verità, va pensata come conoscibile» ${ }^{16}(508$ E $3-4)$;

2.2.3. «la conoscenza e la verità sono belle entrambe, [...] questa Idea è diversa e più bella» (508 E 4-6);

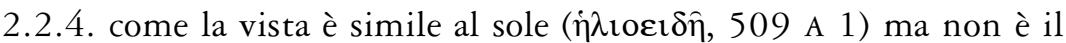

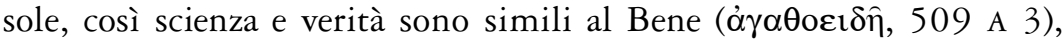
ma non sono il Bene, la cui condizione deve essere giudicata superiore (509 A 4-5).

2.2.5. Come il sole è causa ontologica, oltre che gnoseologica, cioè fornisce agli oggetti anche la generazione, pur non essendo egli generazione (509 B), così deve essere anche per il Bene, che fornisce alle

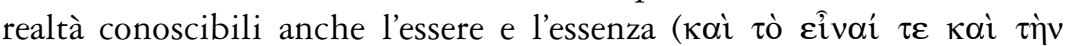
ovoíav, 509 в 7-8), pur non essendo egli stesso interno a questa

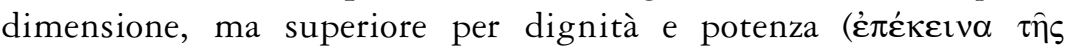

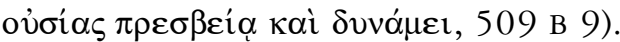

Platone quindi ci ha detto che il Bene è anteriore alla verità e che c'è una sorta di doppio passaggio, con un principio, causa re-

\footnotetext{
${ }^{15}$ Per un necessario approfondimento di questa analogia, basata su tre fattori, e per una discussione di alcune divergenti interpretazioni, cfr. F. FERRARI, L'idea del Bene: collocazione ontologica e funzione causale, in M. VEGETTI (a cura di), Platone. La Repubblica, V, cit., pp. 287-325, partic. pp. 295-303; p. 315 nota 38, e F. CALABI, Il sole e la sua luce, ivi, pp. 327-54, la quale, oltre a inquadrare storicamente l'analogia, ricorda che «è introdotta proprio per appianare difficoltà, per rendere semplice e ovvio ciò che ovvio non è» (p. 352).

${ }^{16}$ Cfr. R.J. FogelIN, Three Platonic Analogies, «Philosophical Review», LXXX (1971) pp. 371-81, p. 372: dobbiamo riconoscere da una parte la trascendenza del Bene rispetto ai processi conoscitivi, dall'altro che solo se il bene è conoscibile l'analogia funziona.
} 
mota ma decisiva, e cause prossime, che agiscono sugli oggetti e sulle funzioni conoscitive, un modello complesso di nessi verticali, che non ha spiegato, una sorta di introduzione al dramma della Caverna. Prima però l'Autore, malgrado le sue "reticenze", da buon maestro che non "lascia solo" il lettore, ci offre uno "schemino" per ricomprendere alcuni elementi del discorso proposto, un aiuto prima di affrontare la Caverna. Tale "schemino" è la Linea.

\section{La Linea}

Socrate afferma che ha lasciato molte cose in sospeso e che molte altre ne tralascerà, anche se cercherà di non ometterne di proposito per quanto è possibile nella situazione presente (509 C 9-10).

La trattazione continua la precedente cui è esplicitamente connessa: si riparte dai due principi prima visti, Sole e Idea del Bene. Invece lo stile cambia radicalmente, perdendo gli elementi immaginifici e dandoci un diagramma matematico.

\subsection{Il diagramma}

I due principi comandano l'uno sul genere e sul mondo intel-

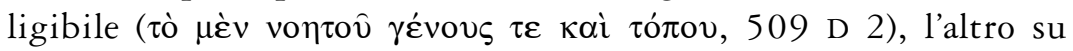

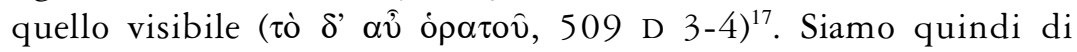
fronte alla formalizzazione della divisione, presentata nell'analogia del Sole, in due ambiti ( $\varepsilon \varepsilon^{\prime} \delta \eta, 509$ D 4), il visibile e l'intelligibile.

Platone dà "un compito" al lettore: disegnare una linea divisa in due parti, precisando che sono diseguali e che la successiva divisione è fatta secondo la stessa proporzione (509 D). Dobbiamo pensare ad una linea costituita da una serie decrescente di segmenti ${ }^{18}$,

${ }^{17}$ La qualificazione di "visibile" viene presentata come casuale, ma poi torna costantemente.

${ }^{18}$ Privilegio le ragioni del numero in uno schema matematico-quantitativo e non c'è dubbio che "salendo" il numero degli oggetti coinvolti decresce fino al Principio primo. Molti sostengono la costruzione contraria, privilegiando una valutazione di tipo assiologico. Ma non mi sembra che l'assiologia platonica sia esente dalla tendenza al re- 
posta in verticale, non solo per ragioni di coerenza con la descrizione fin qui fatta, ma perché Platone parla di parte bassa (кó $\tau \omega, 511$

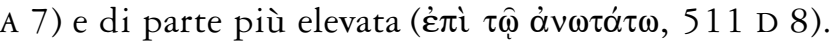

Il testo non dice quale proporzione occorre stabilire tra le parti, segno che non è questo il punto decisivo. D'altra parte, i due segmenti centrali hanno necessariamente la stessa dimensione. Platone non esplicita questo dato, ma non ne ha bisog no, perché egli si aspetta che il lettore faccia questa operazione, cioè disegni la linea, e lo scopra, se già non lo sa, da sé.

C'è stato un grande dibattito tra chi sostiene la causalità di questa struttura, e quindi la sua irrilevanza filosofica, e chi ne cerca la spiegazione. Ma una costruzione "voluta" come questa, in un ambiente attento ai procedimenti geometrico-matematici come l'Accademia, difficilmente può essere casuale. Si tratta di vedere se dal testo scaturisce qualche suggerimento che eviti l'arbitrio dell'interprete.

È certo però la linea presenta una forte verticalizzazione, da un segmento lungo a uno molto più corto. La cosa è logica 1) sul piano ontologico per l'opposizione uno-molti (nella trattazione precedente la caratteristica del mondo visibile è la molteplicità, quella del mondo ideale l'unità ${ }^{19}$ ); 2) sul piano conoscitivo in quanto le parti si differenziano «per un rapporto di chiarezza e oscurità tra loro» (509 D 9), affermazione ispessita dal fatto che la prima divisione del visibile è ricondotta all'opposizione verità-non verità (510 A 8-9).

Nella sezione visibile il primo segmento è costituito dalle immagini. Platone chiarisce che parla delle ombre, dei riflessi negli specchi, nell'acqua e così via. Nell'altro settore vanno invece poste le cose reali, classificate come: animali, vegetali, manufatti.

È quindi facile stabilire uno stacco forte tra l'immagine e l'oggetto di cui è immagine.

stringimento (si pensi, ad esempio, ai piaceri puri, piccoli e rari, eppure più piacevoli, più veri e più belli degli altri, in Phil. $53 \mathrm{~B}-\mathrm{C})$.

${ }^{19}$ Cfr. per es. 476 A, 479 A, 507 A-B. 


\subsection{La sezione intelligibile}

Nella divisione della sezione intelligibile si parla di attività ricondotte all'anima. Questa nel primo segmento è costretta

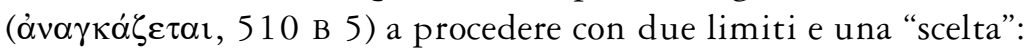

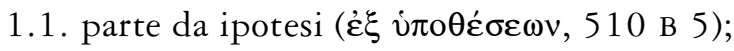

1.2. si serve come di immagini ( imitate nell'altro settore;

2. volge non verso il principio, ma verso la fine (510 в 5-6).

Troviamo qui una indicazione sulle possibili ragioni della identica lunghezza dei due segmenti centrali. L'anima lavora, per così dire, sullo stesso materiale, anche se con un atteggiamento intellettuale del tutto diverso da quello che domina nella sfera del sensibile. Ciò comporta anche oggetti diversi ${ }^{20}$ : nella dianoia si trovano i modelli ontologici di tutte le realtà sensibili che sono nella pistis: animali, vegetali, manufatti.

Quanto al secondo segmento:

1. ha un rapporto originario con un principio anipotetico ( $\tau$ ò $\dot{\varepsilon} \pi$ '

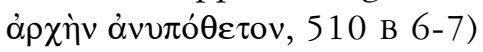

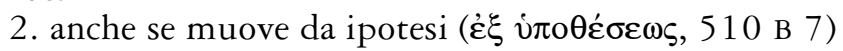

3. senza le immagini relative (510 в 7-8)

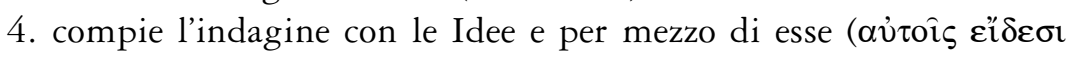

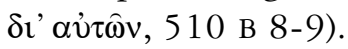

${ }^{20}$ Per J.S. MORRISON, Two Unresolved Difficulties in the Line and the Cave, «Phronesis», XXII (1977) pp. 212-31, l'uguale lunghezza dei due segmenti si spiega perché «nella sezione intelligibile la mente usa gli originali della sezione visibile come immagini; in altre parole, le stesse cose sono trattate due volte, prima, nella sezione sensibile, dai sensi come originali, poi, nella sezione intelligibile, dalla dianoia come immagini» (p. 223). Ma, a parte che lo schema sarebbe "disarmonico" con quattro facoltà e tre oggetti, qui Platone dice solo che l'anima "usa" strumentalmente figure sensibili, ma i suoi oggetti sono diversi: altro è avere come oggetto un disegno che non è un (vero) quadrato, altro è pensare il quadrato in sé. 
A fronte di una esposizione così breve Glaucone dichiara di non aver capito. E ne ha tutte le ragioni! L'unica differenza chiara è che il primo procedimento è legato al sensibile mentre l'altro se ne stacca del tutto. Però, mentre è detto che l'uno discende verso le cose concrete, si deve intuire che l'altro ascende verso il principio, e ancor più difficilmente si può pensare che lo fa per fondare i postulati. Analogamente, mentre è chiaro che il secondo procede per Idee, bisogna ipotizzare che lo stesso vale per l'altro.

Socrate deve quindi riprendere la questione, facendo un esempio chiarificatore, basato sul confronto con i matematici.

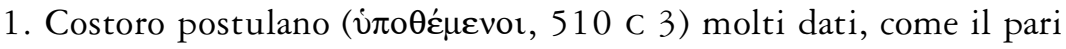
e il dispari e le figure geometriche, cioè pongono gli elementi che strutturano le loro discipline senza giustificarli, quasi fossero pre-

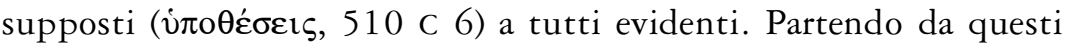
svolgono le loro analisi. In sintesi, la giustificazione della struttura delle matematiche è puramente funzionale: si costruisce un modello che, in forza degli elementi posti, consente calcoli e teoremi.

2. Svolgono ragionamenti utilizzando figure, però non pensano a queste, ma alle forme astratte cui assomigliano, cioè pensano al quadrato in sé e non a quello che disegnano. Ma certo non per caso Platone esplicita che siamo sempre di fronte a tutte le movenze di questa realtà, immagini, cose, Idee: i matematici fanno o disegnano figure, che producono immagini ( come immagini cercando di vedere quelle realtà in sé che uno non può vedere se non con il pensiero» $(510 \mathrm{E} 3-511 \mathrm{~A} 1)^{21}$.

È ora possibile chiarire quello che prima Socrate ha accennato e giustificare la "particolare" caratteristica di questo ambito, quasi un anello di congiunzione tra il puro noetico e il sensibile. Infatti ab-

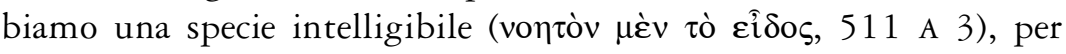

${ }^{21}$ Per evitare confusioni Platone ribadisce i due tipi di immagini: i matematici si servono come immagini (દikóø, 511 A 6) di quelle realtà concrete di cui, nel livello inferiore, si danno immagini e che, rispetto a queste ultime, sono considerate migliori e più chiare. 


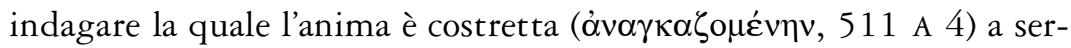
virsi di ipotesi ( $\dot{\pi} \pi \theta \dot{\varepsilon} \sigma \varepsilon \sigma \mathrm{l}, 511 \mathrm{~A} 3$ ) rinunciando a muovere verso il principio in quanto non è capace di trascenderle. Il limite è, al contempo, soggettivo e oggettivo.

Quel che emerge è una conoscenza ingenua, perché ritiene evidenti le scelte che, dal punto di vista dei suoi processi, sono necessarie, e incompleta, perché non tenta di verificare la fondatezza di tali scelte. E tuttavia è pensiero elevato e anche dialogico: basta pensare alle dimostrazioni di un teorema.

Si può ora affrontare l'altro segmento: «Sappi ora che io parlo dell'altra parte dell'intelligibile, quella che il ragionamento stesso attinge con la potenza della dialettica facendo dei postulati non già dei principi, ma veri e propri presupposti, come punti d'appoggio e di partenza, muovendo dai quali giungere a ciò che è anipotetico, al principio del tutto; una volta raggiunto questo, attenendosi poi a ciò che da quello consegue, scendere così verso la fine, senza servirsi assolutamente di nessun dato sensibile ma delle Idee stesse

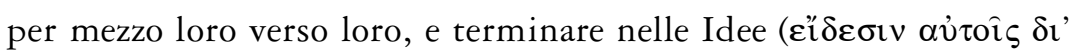

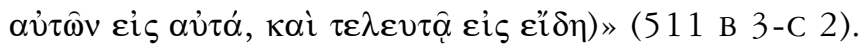

Le differenze sono ora chiare: la noesis non assume acriticamente le ipotesi, ma nemmeno le nega. Il procedimento dialettico di "risalita" le "usa" e nel contempo le fonda, nel senso che non le considera sicure finché non siano dedotte da un postulato precedente. E poiché nel contesto platonico il rinvio all'infinito è impossibile, occorre pensare che questo processo termini in una realtà anipotetica. Socrate enfatizza la presenza delle Idee: siamo nella sfera del puro pensiero che ascende a pensieri ancora più astratti per raggiungere il principio del tutto e poi, con la movenza tipica della dialettica platonica $^{22}$, scende fino alle ultime conseguenze senza utilizzare $i$ dati sensibili.

\footnotetext{
${ }^{22}$ Per la dialettica platonica rinvio ai miei: Dialektik und Prinzipientheorie in Platons 'Parmenides' und 'Philebos', in Platonisches Philosophieren, Zehn Vorträge zu Ehren von H.J. Krämer, herausg. von TH.A. SZLEZÁK unter Mitwirkung von K.-H. STANZEL, Hildesheim-Zürich-New York 2001, pp. 109-54 (trad. it.: Dialettica e Teoria dei principi nel 'Parmenide' e nel 'Filebo' di Platone, "Annali della Facoltà di Lettere e filosofia del-
} 
Ma: 1) non si dice quali sono questi punti ultimi cui il processo discensivo arriva, e 2) si afferma che l'intelligenza (vónбıৎ) risale al principio, che però essendo primo e anipotetico, deve ancora una volta essere sia ammesso tra le cose conosciute, sia riconosciuto nella sua radicale diversità da tutto il resto.

Platone aggiunge altri chiarimenti:

1. la parte dell'essere e dell'intelligibile studiata dalla dialettica è più certa di quella delle tecniche che si basano su ipotesi $(511 \mathrm{C})$; la dialettica, anche in forza del suo oggetto, si stacca dalle altre forme conoscitive;

2. il limite di queste tecniche è che, pur agendo con il pensiero

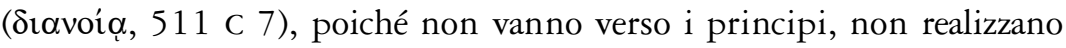
l'intelligenza (vô̂v, $511 \mathrm{D} 1$ ) di quegli oggetti che pure sono noetici ( il difetto è (anche e soprattutto) di rinunciare a salire verso i principi realizzando una superiore forma di conoscenza (il nous);

3. chiama questa conoscenza dianoia considerandola intermedia tra

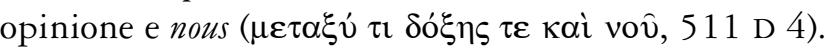

Quindi, mentre nel libro $\mathrm{V}$ la doxa è intermedia fra sapere $\mathrm{e}$ ignoranza, qui - con uno schema diverso - la dianoia diventa intermedia tra doxa e nous. Bisogna quindi prendere atto che Platone usa schemi diversi e che li utilizza con molta libertà, visto che qui si basa su tre soli dati: l'opinione, che corrisponde all'intera prima sezione, la noesis, secondo segmento della seconda sezione, la dianoia, primo segmento della seconda sezione, pensiero ancorato al sensibile, intermedio tra l'intera prima sezione e l'ultimo segmento della seconda. Basterebbe disegnare i due diagrammi, rispettando le differenti lunghezze, per capire quanto sono diversi!

l'Università di Macerata», XXXIV (2000) pp. 55-103); Pervasività e complessità della dialettica platonica, in M. MIGLIORI-A. FERMANI (a cura di), Platone e Aristotele. Logica e dialetti$c a$, Brescia, in corso di stampa. 
Un'ultima osservazione: qui la differenza tra dianoia e doxa si mescola con l'opposizione tra senso e pensiero. Sorge subito una domanda: se l'opinione è sensoriale dove collochiamo un'opinione mentale o un pensiero concreto? Ci torneremo tra poco.

Socrate può ora concludere, presentando i quattro segmenti e le rispettive funzioni dell'anima ( $\pi \alpha \theta \dot{\eta} \mu \alpha \tau \alpha \dot{\varepsilon} v \tau \hat{n} \psi v \chi \hat{\eta}, 511$ D 7), cui quindi tutto viene ricondotto ${ }^{23}$ : la più elevata è la noesis, la seconda la dianoia, la terza è la pistis, la quarta l'eikasia.

Si conferma anche la proporzione tra dimensione ontologica e gnoseologica connessa all'anima: quanta è la verità di cui gli oggetti partecipano, tanta è la certezza di cui partecipano queste funzioni. Troviamo qui un segno dell'azione del Bene, che struttura e dà "misura" all'oggetto: «il differente rapporto og gettivo con il buono degli oggetti spiega il diverso grado di chiarezza che essi consentono all'anima rispetto a se stessi ${ }^{24}$.

\subsection{Il valore conoscitivo della prima sezione}

I risultati di questa analisi ci permettono di tornare sulla prima sezione. Anche se a Platone interessa assai più la seconda (logico, visto che tutto è in funzione della figura del filosofo), la prima sezione non è priva di interesse. Se, seguendo indicazioni diffuse, decidessimo di ignorarla in quanto filosoficamente irrilevante, dovremmo pensare che questa trattazione si stacca del tutto sia da quella precedente del Sole ${ }^{25}$, sia dalla successiva ripresa del libro VII, che, ricapitolando i tipi di conoscenza, richiama esplicitamente questa trattazione (533 D 6-7) e ripropone una quadripartizione delle forme conoscitive (ne accenneremo tra poco).

${ }^{23}$ Una posizione che sarà confermata in Phil. 33 B-34 C.

${ }^{24}$ F. Franco RePellini, La linea e la caverna, in M. Vegetti (a cura di), Platone. La Repubblica, V, cit., pp. 355-403, p. 355 . Questo però, contrariamente a quello che sembra pensare Repellini (ivi, p. 357), fa maggiormente risaltare l'assenza di una chiara determinazione degli oggetti del pensiero nella Linea (cfr. infra, 3.2.).

${ }^{25}$ Invece, è universalmente ammesso che «l'immagine della linea [...] è introdotta come uno sviluppo della similitudine del sole» (F. FRANCO REPELLINI, La linea cit., p. 355). 
Quanto all'eikasia, il primo problema è di traduzione: «qualcosa come "fede nell'immagine" o "dipendenza dalle immagini" rende meglio quel che Platone intende» ${ }^{26}$. Tuttavia, lo spazio riservato alle ombre può sembrare eccessivo: «l'eikasia non sembra corrispondere a niente di significativo nelle nostre vite, ma sembra essere lì per amore dell'analogia fra mondo visibile e mondo intelligibile» ${ }^{27}$. Ma non può essere ignorato il fatto che Platone mantiene sempre questo settore conoscitivo, ben diverso dall'ignoranza, un tipo di sapere che potrebbe forse essere capito in relazione con altre parti della Repubblica ${ }^{28}$.

In tutti i casi, se si pensa che l'eikasia non sia una conoscenza per quanto inferiore 1) in che cosa si distingue dall'ignoranza, dal non sapere? Se in nulla, 1.1.) vedere una cosa allo specchio (o in fotog rafia) o non vederla è lo stesso; 1.2.) una copia sarebbe falsa in assoluto, mentre lo è solo in relazione al "vero" og getto.

D'altra parte, senza questo settore Platone non potrebbe stabilire il duplice nesso $(510 \mathrm{~A})$ :

1) eikasia: pistis = opinabile: conoscibile;

2) immagine: modello = falso: vero.

Il primo rapporto ci riconduce al v libro, quindi all'interno di un diverso schema, e ci porta a dire che l'eikasia è condannata all'interno della relazione con la pistis (così come la doxa rispetto alla scienza) $m a$ non in sé (= non è ignoranza). Analogamente nel secondo rapporto l'immagine è condannata in relazione al modello.

Se invece si dà alle affermazioni platoniche un valore assoluto, a prescindere dalla relazione, il primo rapporto rende impossibile

${ }^{26}$ 'TH.A. SZLEZÁK, La 'Repubblica' di Platone. I libri centrali, a cura di M. Migliori-C. Danani, Brescia 2003, p. 90.

${ }^{27}$ J. ANNAS, An Introduction to Plato's 'Republic', Oxford 1981, $1982^{2}$ (with corrections), pp. 249-50.

${ }^{28}$ Ad esempio, «può essere che questa forma di conoscenza includa le arti imitative, le più lontane dalla verità secondo l'analisi svolta nel libro X» (M. VEGETTI, Introduzione, cit., p. 24). 
qualsiasi ulteriore ridimensionamento della pistis (che qui corrisponde alla conoscenza), cosa invece possibile e necessaria a partire dalla sezione successiva all'interno della relazione così costruita. Nello stesso tempo, risulta evidente che le due relazioni sono proprio "diverse" e non vanno intrecciate, perché se si fa corrispondere qui l'eikasia all'immagine e la pistis al modello (cosa che in altro contesto potrebbe essere accettata) si deve concludere che la pistis è una conoscenza vera!

Infine, se Platone avesse voluto usare la parte bassa della linea solo per esemplificare quella alta, avrebbe potuto creare due linee staccate: «due linee avrebbero meglio funzionato come analogia allo stesso modo in cui nel Sole si distingue fra il visibile e l'intelligibile» ${ }^{29}$.

Ma Platone dice di disegnarne una sola. Non capiva l'indicazione che dava al lettore? Credo, invece, che confermando questa unica linea volesse sottolineare la necessità sia di amare sempre l'intero, sia di ricordare il limite umano che segna anche il filosofo ${ }^{30}$.

\subsection{La Caverna}

La trattazione dovrebbe continuare mostrando come la Linea sia utile nella successiva trattazione della Caverna che completa il

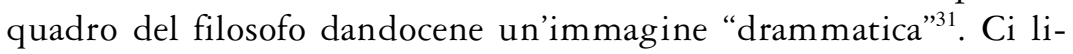
mitiamo a tre osservazioni.

Troviamo nella Caverna lo stesso meccanismo ambivalente visto nella Linea: una scansione forte tra i due mondi, qui nella forma "fuori-dentro", e nello stesso tempo un processo continuo e unitario.

${ }^{29}$ J. ANNAS, An Introduction cit., p. 249.

${ }^{30} \mathrm{Nel}$ Filebo, nella mescolanza per la vita buona, se si hanno le conoscenze superiori, si accettano tutte le altre, anche quella del falso cerchio, perché «è necessario, se vogliamo ritrovare ogni volta la strada di casa» (62 B 8-9), «necessario, se la nostra vita dovrà essere, in qualche modo, una vita» (62 C 3-4).

${ }^{31}$ M. VEGETTI, Introduzione, cit., p. 23: «L'immagine della "linea” traccia un modello statico dell'articolazione dello spazio ontologico ed epistemologico, già accennato nella metafora solare, mentre l'allegoria della caverna rappresenta piuttosto questo spazio come il luogo dinamico di un percorso dell'anima che è insieme etico e intellettuale». 
Si distinguono anche in questo caso quattro forme di conoscenza, sia pure con una significativa correzione ${ }^{32}$. Si conferma il nesso matematiche-dianoia e la natura intermedia di quest'ultima (533 D-E), enfatizzando il rapporto tra episteme e dialettica.

Infine, Platone conferma la conoscibilità del Bene (VII 516 B)

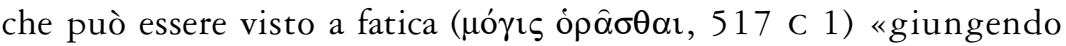
al limite estremo dell'intelligibile» (532 в 1-2). Ancora: «Dunque, così sarà anche per il Bene: chi non è in grado di determinare l'Idea del Bene con il ragionamento distinguendola da ogni altra e, passando come in una battaglia attraverso tutte le prove, desideroso di provare non secondo opinione ( $\kappa \alpha \tau \grave{\alpha} \delta o ́ \xi \alpha v)$, ma secondo l'essere $\left(\kappa \alpha \tau^{\prime}\right.$ o $\left.\sigma \dot{\sigma} \alpha v\right)$, non affronti tutte queste con un suo ragionamento che resti sempre in piedi, di chi sta in tale condizione non dirai che conosce il Bene in sé né alcun altro bene; ma se pur ne coglie una qualche immagine, la coglie con l'opinione ( $\delta$ ó $\xi \emptyset$ ), non con la scienza (غ่ $\pi \imath \sigma \tau \dot{\mu} \mu \eta \emptyset) »$ (VII 534 B 8-C 6).

Qui vorrei solo riflettere sull'uso di doxa. Nel primo caso è contrapposta ad ousia; per ragioni di "coerenza" bisogna dunque forzare uno dei due termini in modo da dire o "non secondo opinione, ma secondo verità" oppure "non secondo la realtà diveniente, ma secondo quella stabile". Credo che questo "gioco" per Platone non fosse scandaloso, perché le due affermazioni gli apparivano, se non equivalenti, certo non molto diverse. Nel secondo uso, doxa si oppone ad episteme, per cui potrebbe anche rientrare nel nostro schema, se non fosse per il fatto che l'attività implicata sembra mentale e non sensoriale. Ciò ripropone un problema non più rinviabile.

\section{Alcuni necessari chiarimenti}

\subsection{La 'dianoia' e le matematiche}

Platone esemplifica la dianoia con le matematiche. Tuttavia, nulla ci obbliga a pensare ad una loro identificazione, ad esclusione

${ }^{32}$ Cfr. quanto dico in M. Migliori, Sul Bene cit., pp. 134-5. 
delle altre tecniche e forme conoscitive. Al contrario, il nesso tra la pistis e la dianoia ci ricorda che in quella sfera si parla di cose, cioè vegetali, animali e manufatti. La dianoia ha a che fare con le Idee (applicate alla dimensione concreta).

Se così non fosse, dovremmo concludere che

1. esistono solo le matematiche e la dialettica, e la classificazione delle scienze teoriche e pratiche che ci propone, ad esempio, la diairesi del Sofista e del Politico sarebbe priva di senso;

2. dovremmo ammettere l'esistenza solo di enti matematici, mentre la dialettica delle Idee è qui proposta e riproposta.

Questa convinzione, minoritaria, che la dianoia non coincida con il pensiero matematico, non mi fa però accettare la posizione opposta, quasi che la scelta dell'esempio sia casuale se non sbaglia$\mathrm{ta}^{33}$. A me sembra, invece, che qui troviamo accennata la trattazione delle matematiche che sarà svolta nel Filebo, 56 C-58 A: c'è l'aritmetica dei più, che si rivolge alle cose disomogenee, e quella dei filosofi, che applicano la matematica a realtà omogenee, cioè ai numeri stessi. L'aritmetica, la logistica e la metretica sono doppie, a seconda che si vedano nel momento applicativo o nel momento strutturale. Quindi, per tornare alla Linea, abbiamo numeri nella pistis (utilizzati nella nostra quotidianità), numeri nella dianoia (delle matematiche) e numeri nella noesis, che portano verso il principio. E sono tre "numeri" molto diversi tra loro.

${ }^{33}$ Questa mi sembra la posizione di F. TRABATTONI, Il sapere del filosofo, in M. VegetTI (a cura di), Platone. La Repubblica, v, cit., pp. 151-86, pp. 159-60: «non bisogna dare troppa importanza a questo motivo né tanto meno usarlo come punto di vista privilegiato per comprendere il terzo segmento. Infatti, le matematiche sono introdotte come esempio per chiarire quanto Socrate dice all'inizio, in 510b4-9, per cui si deve ritenere che in questo passo ci sia già tutto quanto è essenziale all'argomentazione». Ma l'esempio serve proprio a chiarire ciò che nella prima formulazione può essere frainteso. Infatti Glaucone non capisce e chiede spiegazioni, $i d$ est Platone ritiene che sia necessario dire qualcosa di più al lettore. 


\subsection{Alcuni limiti della Linea}

Siamo forse ora in grado di giustificare la nostra "brutale" presentazione della Linea come uno "schemino".

Il diagramma ha una serie di sbilanciamenti. La differenza tra le due sezioni è di ambito, uno legato alla dimensione sensoriale (il visibile), l'altro alla sfera psichica (il pensabile). Le differenze tra i segmenti sono differenze di chiarezza, ma anche opposizioni di vero-falso e/o di fondato-infondato. Inoltre, i primi due segmenti hanno differenze di contenuto (immagini-oggetti), gli ultimi due di procedimento (vincolato al sensibile e discensivo-libero e ascensivo). Pertanto, la questione dell'oggetto dei due segmenti finali rimane quantomeno dubbia. Se ad esempio ci si pone la domanda: "dove sono l'Idea del pari e del dispari come struttura base dei numeri?", si dovrebbe rispondere che sono sia nella dianoia sia nella noesis, a seconda dell'uso che il pensiero ne fa. Ma è una "deduzione", perché il testo "tace". O forse, sottolineando la natura di intermedio della dianoia e presentando l'esempio matematico, Platone accenna "in modo cieco" (cioè incomprensibile al lettore "ignorante") un problema di grande rilievo teorico ${ }^{34}$.

Inoltre, dobbiamo riconoscere che qui abbiamo solo "uno schema" e non "lo schema". In effetti, abbiamo intravisto vari elementi, nel testo, che fuoriescono dal quadro (anzi, a partire dal libro $\mathrm{v}$, abbiamo visto due schemi diversi, triadico e binario).

Se poi prendiamo le mosse dal testo, dal matematico che non pensa al quadrato che disegna, ma al quadrato in sé, e ci chiediamo:

${ }^{34}$ Su questo riflette TH.A. SZLEZÁK, La 'Repubblica' cit., pp. 91-6, per il quale non è corretto ignorare che le "idee matematiche" (molteplici) sono diverse dall'Idea (unica). Pertanto, parlando di quadrato in sé non si accenna in modo generico a tutte le Idee, ma ai numeri ideali, coglibili solo con la dianoia. Ma se si irrigidisce questa condivisibile osservazione, si aprono altri problemi: il rapporto pistis-dianoia, l'affermazione platonica che

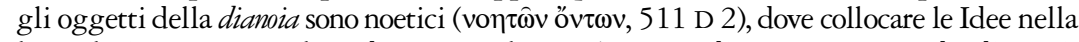
loro relazione non con altre Idee ma con le cose (un procedimento, a suo modo, discensivo). Credo, quindi, sia meglio accettare i "limiti" della Linea rispetto al complesso mondo teorico di Platone. Szlezák stesso sostiene che, invece di cercare di ridurre il «numero dei tipi di oggetti ontologici, ci si sarebbe dovuti domandare con assai maggiore ragione se tale numero non vada invece ampliato». Condivido, ma questo implica una ancora maggiore insufficienza del nostro schema. 
"dove collochiamo il pensiero del quadrato?", scopriamo che in questo schema non c'è risposta.

Se infatti lo collochiamo nella pistis, in forza dell'oggetto e per rispettare la contrapposizione stabilita da Platone, 1) la pistis si divide in due, perché abbiamo la visione del quadrato e il pensiero del quadrato; 2) lo schema mostra delle crepe, perché non siamo nella sfera del (visibile) sensoriale, ma in quella del pensiero.

Se però diciamo che è pensiero 1) non rispettiamo la lettera del testo; 2) se insistiamo a porlo nella dianoia 2.1.) dobbiamo dividerla in due funzioni diverse; 2.2.) non potremo dire che questo (tipo di) pensiero sia un'opinione, perché la doxa si ferma alla pistis, è solo sensoriale.

Quest'ultimo è un problema centrale, tanto più che, come ricorda Ferrari, almeno in due passi Platone sembra affermare che «di una forma intelligibile si può avere anche opinione» ${ }^{35}$. Vorrei non essere frainteso: sono certo che in Platone c'è un pensiero che è opinione, vera o falsa; dico però che questa opinione in questo schema non può trovare collocazione. Ma nel testo stesso, ovviamente, si avverte a volte la possibilità/necessità di una diversa semantizzazione della doxa.

\subsection{Approccio teorico e storico}

Questo riferimento al testo è importante perché spesso i problemi nascono dalla sovrapposizione di nostre distinzioni concettuali a un discorso "banale" di Platone. Non voglio dire che non si può "interrogare" un testo, ma che in chiave storica bisogna rispettare la consapevolezza teorica del pensiero antico. Per tornare a una questione già accennata ${ }^{36}$, a mio avviso Platone ragiona in questo modo: posso affermare la verità di una proposizione, perché in questa un predicato si riferisce a un soggetto, cosa possibile perché il soggetto della proposizione esiste. Se infatti non esistesse non sarebbe possibile la predicazione e quindi ogni discorso sulla verità sarebbe un nonsense. Se si accetta questo, molte questioni poste dal-

\footnotetext{
${ }^{35}$ F. FERRARI, Teoria cit., p. 401 . I due passi sono 505 B, 506 B.

${ }^{36}$ Cfr. supra, nota 5 .
} 
la critica, pur non perdendo la loro valenza teorica, non sono più "platoniche".

Analogamente, credo che sia un'operazione solo teoretica chiedere se alcune distinzioni, per es. tra opinione e conoscenza, siano fondate solo soggettivamente o solo oggettivamente, perché questa alternativa non si pone. Come abbiamo visto ripetutamente, Platone passa tranquillamente da uno all'altro ambito: "conoscenza e opinione si distinguono per la loro struttura epistemica intrinseca, l'una essendo infallibile, l'altra contenendo in sé la possibilità dell'errore. Ma tutto ciò non preclude la possibilità di individuare la ragione di questa differenza a latere objecti, vale a dire nella natura dell'oggetto intenzionato, la quale garantirebbe l'infallibilità della forma cognitiva a esso relativa» ${ }^{37}$.

Più in generale, "la "linea" istituisce dunque, com'è consueto in Platone [corsivo mio] un rapporto bicondizionale fra livelli di conoscenza e livelli di realtà: se vi sono diversi gradi di essere (che significa anche esser-veri) degli enti, allora vi sono diversi gradi di conoscenza; ma vi sono diversi gradi di conoscenza (in primo luogo "opinione" e "scienza", doxa ed episteme); dunque vi sono diversi gradi di realtà» ${ }^{38}$. Questo modo platonico di ragionare può apparire insufficiente o errato, sulla base di un giudizio teorico, che va tenuto distinto dal tentativo di riprodurre, per quanto è possibile, il pensiero del filosofo ateniese.

\subsection{Un gioco di ambivalenze}

Possiamo cercare di trarre qualche conclusione.

Non può essere sottovalutato il fatto che qui Platone dice, e dice anche molto, ma non argomenta. Può farlo perché sceglie il procedimento per immagini, e non per ragionamenti. Si tratta di una scelta comprensibile, in quanto 1) voleva sostenere una tesi che doveva apparire paradossale; 2) per questo doveva per la prima volta presentare con chiarezza la figura del filosofo, distinguendolo dai

\footnotetext{
${ }^{37}$ F. FERRARI, Teoria cit., p. 404.

${ }^{38}$ M. VEGETTI, Introduzione, cit., p. 23.
} 
filodossi e da quelli che egli considera pseudofilosofi, "accennando" quindi a temi di grande rilevanza e difficoltà.

Proprio per questa sua scelta credo sia meglio non porre al testo domande teoriche forti. Mi limiterò quindi a elencare alcuni dati che sono emersi, anche se questo modo di procedere, che svela la presenza nel testo di espliciti nuclei problematici, rischia di lasciarci insoddisfatti e "pensierosi". Ma ritengo che questo fosse proprio uno degli scopi che Platone si riprometteva.

Schematicamente, nel testo abbiamo trovato che:

1.1 chi ama il sapere ne ama tutti gli aspetti, non uno solo; 1.2 . in senso proprio, solo l'episteme è vero sapere;

2.1. la doxa è diversa dal non sapere; 2.2. rispetto al vero sapere è cecità;

3.1. i segmenti della Linea sono tanto staccati da poter anche essere contrapposti come vero-falso; 3.2. ci sono vicinanze tali da indurre, in certi casi, quasi a identificarli;

4.1. con queste immagini Platone apre ampi squarci sulla sua concezione filosofica; 4.2. su pressoché nessuno dei punti più importanti dà risposte chiare e conclusive;

5.1. la Linea ci offre uno schema onto-epistemico; 5.2. emerge nel testo stesso (oltre che in una riflessione "elementare") la necessità di altri schemi, non coincidenti con questo;

6.1. Platone rimanda ad un assoluto, al limite estremo dell'intelligibile; 6.2. più volte ci ricorda il limite della natura umana e la necessità di attingere l'assoluto per quanto è possibile;

7.1. il Bene deve essere conosciuto; 7.2. la sua natura superiore a tutto rende problematica la definizione di questo tipo di conoscenza; 8.1. percorrendo la Linea si arriva al Bene; 8.2. Il Bene non può essere nella Linea, perché è oltre l'essere e l'episteme ${ }^{39}$.

\footnotetext{
${ }^{39}$ Questo vale per il Bene, Idea "estrema" e particolarissima, ma non può essere esteso alle altre Idee, negando quello che il testo dice sulla dialettica e rendendo incomprensibile il concetto stesso di episteme.
} 
Se una qualsiasi interpretazione pretende di ricondurre a unità queste tensioni, non può che infilarsi in un vicolo cieco, dando luogo a un dibattito infinito perché l'ermeneutica unilateralmente proposta, quale che sia, si trova esposta all'evidenza di affermazioni che la contraddicono.

In realtà, troviamo qui un segno della differenza tra il nostro modo di "fare filosofia", figlio delle idee "chiare e distinte" e teso a costruire modelli alternativi (aut ... aut), e quello classico, soprattutto platonico-aristotelico, che cerca di giustapporre, con un costante pluralismo metodico, schemi diversi (et ... et) per capire una realtà intrinsecamente uni-molteplice. Ciò consente affermazioni perentorie, subito "mitigate" da altre che ricordano altri aspetti altrettanto reali.

Così il filosofo-dialettico resta un essere umano che ha esperienza come gli altri di tutte le cose concrete, senza le quali non potrebbe reggere la città. Certo, quello che lo caratterizza non è questo tipo di conoscenza, per quanto affinata. Bisogna dunque sempre precisare che, non in quanto Socrate, individuo concreto, ma in quanto filosofo preso in sé (anche se concretamente presente in un individuo), il filosofo non desidera conoscere il mondo sensibile, ma quello superiore. Ciò non lo porta in un'altra dimensione, perché quel sapere è "utile" alla sua vita felice come è "utile" per la costruzione di uno Stato.

E ciò spiega perché la separazione delle sezioni e dei segmenti non distrugge l'unitarietà della Linea.

\subsection{Tre brevi osservazioni conclusive}

In primo luogo, a me sembra una scelta corretta ed efficace che, a fronte di un'impresa così complessa, Platone abbia puntato sulle immagini in modo da dire molto, ma non troppo, mantenendo un livello di comprensibilità elevato, come mostra il successo della Repubblica.

In secondo luogo, Platone ci ha fatto vedere che non condanna i livelli inferiori, a condizione che ci siano quelli superiori. La conoscenza delle cose belle va bene se c'è la conoscenza delle Idee del bel- 
lo; analogamente il procedimento della dianoia non è da condannare, se si fa operare anche la noesis ${ }^{40}$.

Infine, Platone "onestamente" ha fatto dire a Socrate che avrebbe omesso molte cose. Perché allora ci si stupisce delle "mancanze" e "imprecisioni"? Credo che sia perché non si riconosce che Platone scrive con un intento più educativo che comunicativo ${ }^{41}$. La Linea serve ad aiutare il lettore a non perdersi in questi libri, con uno schema non perfetto e non completo, ma utile per la riflessione autonoma di un lettore disposto a pensare sul testo. E in effetti l'ermeneutica platonica mostra quanto ci sia (e quanto manchi) in questi libri.

Non volendo però aprire una discussione sulla scrittura platonica $^{42}$, cito la posizione equilibrata di Ferrari: "proprio il carattere generale della teoria platonica della comunicazione si oppone alla pretesa di trovare esposta [...] la totalità dei teoremi filosofici relativi all'ontologia e all'epistemologia. In verità, Platone si limita a presentare quelle dottrine che gli sembrano adattarsi allo scenario

\footnotetext{
${ }^{40}$ In questo senso mi sento di accettare la proposta di F. FRANCO REPELLINI, La linea cit., p. 361, di considerare i due segmenti della seconda sezione sia in parallelo sia in serie.

${ }^{41}$ Su questo terreno, si stanno muovendo ormai molti studiosi di diversa ispirazione. Ad es., D. SEDLEY, The Midwife of Platonism. Text and Subtext in Plato's 'Theaetetus', Oxford 2004, a proposito del Teeteto, riprende un' intuizione di Burnyeat per stabilire «la distinzione tra dialettica interna al dialogo e dialettica tra testo e lettore» (p. 6). Letto in questa chiave "maieutica", il "fallimento" conclusivo è apparente e voluto perché «non appena Platone porta noi, i lettori, per quanto è possibile vicini alla vera definizione, non appena è ad un soffio dal dichiararlo, il suo lavoro è compiuto. Tocca poi a noi, ai lettori» (p. 5); «dal momento che la prima regola della maieutica filosofica non è di fornire la risposta giusta agli interlocutori, ma di renderli capaci di generarla da sé grazie alle loro risorse interiori, il dialogo deve inevitabilmente interrompersi prima di fornirci la risposta corretta alla sua domanda principale. Da ciò non consegue che Platone non la conosca» (p. 11).

${ }^{42}$ Cfr. i miei Tra polifonia e puzzle. Esempi di rilettura del "gioco" filosofico di Platone, in G. CASERTANO (a cura di), La struttura del dialogo platonico, Napoli 2000, pp. 171-212; Comment Platon écrit-il? Exemple d'une écriture à caractère "protreptique", in M. FATTAL (éd.), La philosophie de Platon, II, Paris 2005, pp. 83-118 (trad. it.: Come scrive Platone. Esempi di una scrittura a carattere "protrettico", "Annali della Facoltà di Lettere e filosofia dell'Università di Macerata», XXXVII (2004) pp. 249-77).
} 
drammatico e al contesto teorico raggiunti in quel preciso momento dello sviluppo del dialogo ${ }^{43}$.

Se gli studiosi di Platone si sono "avventati" su questo piccolo "schema" è perché il gioco scritto di Platone è così intrigante che ci si attacca a ogni appiglio per "fare chiarezza". Ma la plurisecolare esegesi platonica mostra che il risultato è esattamente l'opposto. Forse sarebbe meglio prendere atto che Platone non dice mai tutto quello che potrebbe, ma come «il signore, di cui è l'oracolo che si trova a Delfi, non dice né nasconde, ma allude» ${ }^{44}$.

${ }^{43}$ F. FERRARI, Teoria cit., p. 369.

${ }^{44}$ D.-K. 22 в 93. 